Bull. Mater. Sci., Vol. 4, No. 2, April 1982, pp. 75-83. ( Printed in India

\title{
Methods of low gravity simulation
}

\author{
J M HAYNES \\ Department of Chemistry, University of Bristol, Bristol, U.K. \\ MS received 17 June 1980
}

\begin{abstract}
The dependence on gravity forces of both static and dynamic fluid behaviour is explained, and dimensionless numbers are defined which express the relative influence of gravitational and various other forces. Methods of reducing gravitational effects are outlined, including neutral buoyancy, free fall and orbital flight and their relative merits, in studies of various types of fluid behaviour, are compared.
\end{abstract}

Keywords. Gravity neutral buoyancy; free fall; orbital flight; capillarity; convection.

\section{Introduction}

The earth's gravitational field is a very familiar feature of the terrestrial environment : it aperates on us, and our earthly surroundings, with such constancy and near-uniformity, that we may sametimes forget its existence. When we set down a glass of water on the table-top, for example, we expect it to remain there, and we expect the water to remain at the battom of the glass. Yet the ideas expressed by such words as "top" and "bottom", ar "up" and "dawn", awe their meaning to the sense of vectorial direction that the earth's gravitational field imposes on our everyday life.

In recent times, we have seen films of astronauts floating about in a condition of apparent weightlessness : an object can no longer be put "down", but if released will either remain stationary or continue in a state of uniform linear mation, as required by Newtonian mechanics. Water may no longer remain in the "bottom" of a glass, but will take up a configuration dictated by capillary forces-a fact of importance in the design of liquid fuel tanks for spacecraft, as well as in the daily routine of astronauts in need of a drink.

We can, of course, experience an approximation to this state while floating in a swimming bath, although different dynamic behaviour is observed because of the viscosity of the surrounding medium. A free-fall parachutist may experience a similar brief sensation of weightlessness, though he will eventually reach a terminal velocity at which the gravitational force is balanced by viscous drag.

These examples typify some of the ways in which low-gravity conditions can be simulated for experimental purposes, and at the same time they illustrate some of their limitations. 


\section{Gravity-dependent phenomena}

An object of effective mass $m$ at the earth's surface experiences a gravitational force $P$ given by

$$
P=m g,
$$

where $g$, the gravitational acceleration, is defined as a constant having the value $9.80665 \mathrm{~m} \mathrm{sec}^{-2}$. In general, two objects of mass $m_{1}$ and $m_{2}$ separated by a distance $r$ experience an attractive gravitational force of $G m_{1} m_{2} / r^{2}$, where $G$ is a universal constant. $g$ is thus simply $G$ multiplied by the mass of the earth and divided by the square of its radius.

In general, the actual acceleration at a given point will not equal the defined value of $g$, but will vary because of the non-sphericity of the earth, and variations in local topography and density of the underlying rocks. In addition, the measured value of $\boldsymbol{g}$ will be decreased by the centrifugal force arising from the earth's rotation, which varies with distance from the rotational axis, i.e., with latitude.

The effective mass in equation (1) is the true mass in vacuo, corrected for buoyancy; thus, if $v$ is the volume of the object, and $\rho$ and $\rho$ ' the density of the object and the surrounding medium, then the effective net force is

$$
P_{\text {eft }}=v\left(\rho-\rho^{\prime}\right) g .
$$

In comparison with intermolecular and intramolecular forces, the earth's gravitational force is very weak : that is to say, over distances of the order of the range of intermolecular forces, the gradient of gravitational potential is negligible. This means, therefore, that molecular properties of matter are generally unaffected by gravity to any detectable extent. For example, the vertical variation of surface tension $\sigma$ in the gravitational field is given by

$$
\mathrm{d} \sigma / \mathrm{d} z=g \Gamma,
$$

where $z$ is the vertical co-ordinate along which the acceleration $g$ operates, and $\Gamma$ is the surface excess of material in the interface (exprcssed in units of mass per unit area). Inserting reasanable numerical values shows that the surface tension variation aver a vertical height of $1 \mathrm{~cm}$ is only a few parts per million at most, and will thus be some arders of magnitude below the limits of practical detectability. Other properties, such as density, contact angle, diffusion coefficient and viscosity, are similarly insensitive to gravity, and are therefore nat expected to change perceptibly when the effects of gravity are remaved or otherwise varied. (A most important exception occurs in the case of tuids near the critical point, where the compressibility coeffioient tends to infinity. In this case, the vertical variation of density in the gravitational field is significant, and this is a serious impediment to terrestrial observations of critical phenomena).

On the other hand, there are many general phenomena on which gravity has an important influence. . Amang these may be mentioned the equilibrium shape and stability of capillary surfaces, buayancy-driven convection, sedimentation of solid particles in fluids, and the spreading of liquids on solid surfaces. Such phenomena are often af major importance in the processing of materials, and it is for this reason that there is a strong interest in investigating such processes in the absence of gravity. Mareover, the rale played by gravity in such phenomena is 
sometimes rather complex, and the need for simplification, both in experiments and in their interpretation, provides another, mare fundamental motivation for performing studies in the absence of gravity.

In discussing gravity-dependent phenomena, the impartance of gravity relative to other forces is conveniently expressed by an appropriate dimensionless number. For example, the static equilibrium shape of a free liquid surface is controlled by the interplay of gravitational and capillary forces, whose relative magnitudes are given by the recipracal of the Bond number :

$$
(\mathrm{Bo})^{-1}=g R^{2} \triangle \rho / \sigma,
$$

where $\sigma=$ surface or interfacial tension,

$\Delta \rho=\rho-\rho^{\prime}=$ density difference between the twa fluids separated by the interface and

$R=$ some characteristic linear dimension of the system.

We may note here that it is often convenient, in discussing capillary statics in a gravity field, to reduce lengths to dimensionless form (compare Boucher and Evans 1975) by division by the capillary constant

$$
a=(\sigma / g \triangle \rho)^{1 / 2} .
$$

Thus, comparing (4) and (5), systems of small reciprocal Bond number are shown to be those whose characteristic length is small comparcd with the capillary constant :

$$
R \ll a \text { when }(\mathbf{B} 0)^{-1} \ll 1 .
$$

Other dimensionless numbers characterise capillary behaviour in ather kinds of field. Thus, the equilibrium shape and stability of an axisymmetric fluid mass in steady rotation about its axis is governed by the rotational Bond number, $\mathbf{B}_{R}$ :

$$
\mathrm{B}_{\mathrm{O}_{R}}=\sigma / \Omega^{2} R^{3} \Delta \rho,
$$

where $R$ is the radius of gyration of the fluid mass about its axis, and $\Omega$ its angular frequency of rotation. When $\left(\mathrm{BO}_{R}\right)^{-1}$ is much smaller than unity, the interfacial configuration of the fluid mass is not significantly affected by rotation.

In buoyancy-driven convection, the potential energy of a density gradient in the gravitational field is transformed to a kinetic energy of flow, and eventually dissipated by viscous forces. When the density gradient is parallel but opposed to the gravity vector (i.e. denser fluid at the top), the system remains metastable up to a certain critical point; any further increase in the density gradient leads to instability, and convective motion begins. The relevant dimensionless number here is the Rayleigh number, which may be thermal or solutal according to the source of the density gradient :

$$
\begin{aligned}
& \mathrm{Ra}_{\text {therm }}=\left(g R^{3} / v\right)(\beta \Delta T / \kappa), \\
& \mathrm{Ra}_{\mathrm{so1}}=\left(g R^{3} / v\right)(\alpha \Delta c / D),
\end{aligned}
$$

where $v=$ kinematio viscosity, $\beta=$ thermal expansion coefficient, $\alpha=$ solutal densification coefficient, $\triangle T \mid R=$ temperature gradient, $\Delta c \mid R=$ concentration gradient, $k=$ thermal diffusivity and $D=$ diffusion coefficient. Critical values of the Rayleigh number, beyond which unstable convection supervenes, are 
known for a few simple geametries. For example, for a fluid contained between horizontal plates of separation $R$ and of lateral extent much greater than $R$, $(\mathrm{Ra})_{\text {crlt }}=1708$. For higher values of $\mathrm{Ra}$, a more-or-less regular pattern of convective vortices (Bénard cells) may be formed.

When a camponent of density gradient exists normal to the gravity vector, a circulatory convective flow will appear, with a mean fluid velocity $u$ of the order of

$$
u=(v / R) \sqrt{\mathrm{Gr}} \quad(\text { for } \mathrm{Gr}>1),
$$

where the Grashof number is

$$
\mathrm{Gr}_{\text {therm }}=\beta \Delta T\left(g R^{3} / v^{2}\right),
$$

or

$$
\mathrm{Gr}_{\mathrm{sol}}=a \Delta c\left(g R^{3} / v^{2}\right),
$$

according to whether the density gradient is engendered by a gradient of temperature or of solute concentration.

In general, then, we see that in considering the various phenomena that are influenced by gravity, the relative importance of that influence is expressed by means of a dimensionless number, such as $(\mathrm{B} \cdot)^{-1}$, Ra or Gr. Other such numbers can be constructed for other phenomena. The effects of gravity are then minimised by making the appropriate number numerically small. (In the particular case of unstable convection, which displays a threshold, the phenomenon is actually eliminated by reducing the value of $\mathrm{Ra}$. below the value of $(\mathrm{Ra})_{\mathrm{crit}}$ appro-priate to the geometry concerned).

To take a particular example, if a drop of water of $5 \mathrm{~mm}$ diameter is suspended from a horizontal surface under terrestrial gravity, the reciprocal Bond number is about $3 \cdot 4$, and gravity forces are rather more important than surface tension in determining its behaviour. Indeed, it is a matter of cammon experience that drops of roughly such a size will become unstable and detach from the solid surface (although the conditions that govern the onset of instability, and the volume of the liquid that actually detaches, are rather complicated). The effects of capillarity can be made to dominate those of gravity by making $\left(\mathrm{Bo}_{0}\right)^{-1}$ numerically small. This can be accomplished by
(a) reducing $g$,
(b) reducing $R$,
(c) reducing $\triangle \rho$,
(d) increasing $\sigma$.

Conducting experiments "in space"-a term that will be amplified in the next section-corresponds to case (a). Working with systems of small dimensions (case (b)) is often feasible ; in the example considered above, reducing the dimensians of the water drop by a factor of 10 reduces $\left(B_{0}\right)^{-1}$ to 0.03 , and this is reflected in the much mare nearly spherical shape of such a small drop. Case (c) has been explaited for about a century in the so-called Plateau methad of neutral buoyancy : the liquid under study is surrounded by another, immiscible with it 
and of equal density. Case (d) is not of practical importance : pure water has the highest surface tension of non-metallic liquids $\left(72 \mathrm{~m} \mathrm{~N} \mathrm{~m}^{-1}\right.$ at $\left.25^{\circ} \mathrm{C}\right)$, and althou gh liquid metals may have surface tensions an order of magnitude greater, their densities are also greater by a comparable factor.

It should be noted that the length $R$ does nat appear to the same power in the various dimensionless groups. Thus, the different phenomena do not scale with length in the same way, and the advantage of working with small systems (case (b) above) may be lost when different fluid-dynamic effects are coupled, as often happens.

\section{Simulation of low gravity}

\subsection{Free fall methods}

Gravitational attraction is a truly universal force, and although there may conceivably be points in the universe at which the gravity force emanating from all masses is approximately balanced in all directions, there will in general be a net force in one particular direction. Even if such null points in the general gravitational field could be discovered, they would be impossibly remote : they would certainly le well outside the solar system, as the mutual perturbation of planetary orbits demonstrates. (The reader is reminded of the discovery of Neptune and Pluto as a result of observed irregularities in the orbit of Uranus).

There are, however, several methads of avoiding the efiects of gravitational acceleration in an experimental system. The most immediate, perhaps, is to drop the system ; in free fall, all parts of the system are accelerated equally (provided that rotation is absent), and weightlessness prevails. (The low value of $(\mathrm{Bo})^{-1}$ achieved in this way is, of course, the reason for the spherical form of lead shot prepared by the traditional method in a shot tower.) There are several disadvantages : for precisely weightless conditions, the system must fall through an evacuated space to avoid the residual effective acceleration which could be felt if air resistance impeded free fall ; an "observer" (preferably inanimate!) must accompany the experiment, and be retrieved undamaged at the end ; and the duration of free fall is limited by the available vertical height. Nevertheless, drop tower facilities are available in several countries, offering periods of up to $5 \mathrm{sec}$ in free fall.

More extended free fall may be attained by flying an aircraft in a ballistic trajectory. Once again, air resistance interfercs, and must be compensated by application of thrust. Because of the nonlinear speed/drag characteristics of aircraft, this poses quite a difficult problcm for the pilat, but in large aircraft there is no need for a precise solution to be found. Instead, the experiment can be allowed to float freely within the fuselage, while the aircraft is flown within an envelope of parabolae surrounding the intended true trajectory and of crosssection determined by the available space within which the experiment may float inside the aircraft without obstruction. Depending on the maximum speed and power available, weightlessness can be maintained for up to about $45 \mathrm{sec}$, and a human observer can be carried with the experiment.

Sounding rackets provide still more extended periods of frce fall during the period, generally of the order of 5-10 min, of coasting flight outside the dense 
atmosphere. The vehicle must be stabilised against rotation about all the three axes, and the experiment is constrained by limitations of volume, mass and power, and by the need for full automation, with all results being recovered by telemetry. Nevertheless, many valuable experiments have been conducted by this means.

Many experiments demand more prolonged periods of weightlessness than any of the foregoing methods provide, and this can be achieved in so-called "space vehicles", whether manned or un-manned, and whether in near-earth arbit or in interplanetary flight.

\subsection{Orbital and interplanetary flight}

When in a stable orbit around a gravitationally-attracting body such as the earth, the gravitational and centrifugal forces acting on an object are equal and opposed. If atmospheric drag is negligible, an orbiting point mass is then free of net a.ccelerations. For an orbiting body of finite size, rotation about its own centre of mass can produce non-zero resultant accelerations at points away from the centre of mass. Moreover, when such a body maves in a non-uniform gravity field, whether due to orbital ellipticity, in homogeneity of the gravitationally-attracting mass, or influence of ather bodies such as sun and moon, the consequent orbital perturbations will a.lso induce small accelerations at positions away from the centre of mass.

The gravitational attractive force $F_{g}$ between two bodies of mass $m$ and $M$ is

$$
F_{g}=G \frac{m M}{r^{2}},
$$

where $r$ is the distance between their centres of mass, and $G$ is a universal constant with the value $6.66 \times 10^{-8} \mathrm{~cm}^{3} \mathrm{~g}^{-1} \mathrm{sec}^{-2}$. If ane of the bodies is earth, we can substitute its mass and radius $\left(r_{0}\right)$ for $M$ and $r$, obtaining

$$
F=m g_{0},
$$

where $g_{0}=G M / r_{0}{ }^{2}=9.81 \mathrm{~m} \mathrm{sec}{ }^{-2}$ is the acceleration due to gravity at the earth's surface.

We can now calculate the acceleration $g$ at an altitude $h$ above the earth's surface from

$$
g=g_{0}\left(r_{0}+h\right)^{2} / r_{0}{ }^{2} .
$$

We see (figure 1) that $g$ remains significant at large distances from the earth, emphasising the fact that an orbital flight does not represent an escape from the earth's gravity, but only a circumvention of it.

When a space vehicle is in orbit around the earth, $M \gg m$, we can therefore neglect the slight perturbation af the motion of the earth's centre of mass. Considering a circular arbit for simplicity, af radius $r_{0}+h$, the balance of gravitational and centrifugal forces on the orbiting bady yields

$$
m g=m\left(r_{0}+h\right) \omega^{2},
$$

where $\omega$ is its angular velocity. Hence, if $\tau$ is the orbital period,

$$
\text { or } \quad \begin{aligned}
\tau & =\left(2 \pi / r_{0}\right)\left[\left(r_{0}+h\right)^{3} / g_{0}\right]^{1 / 2}, \\
\tau & =2.74 \times 10^{-5}\left(r_{0}+h\right)^{3 / 2} \text { hours, }
\end{aligned}
$$




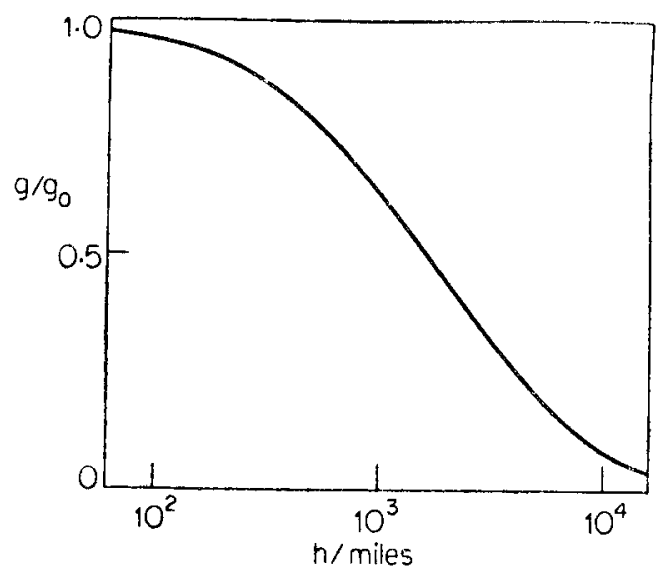

Figure 1. Relative reduction in gravitational force as a function of height above the earth's surface.

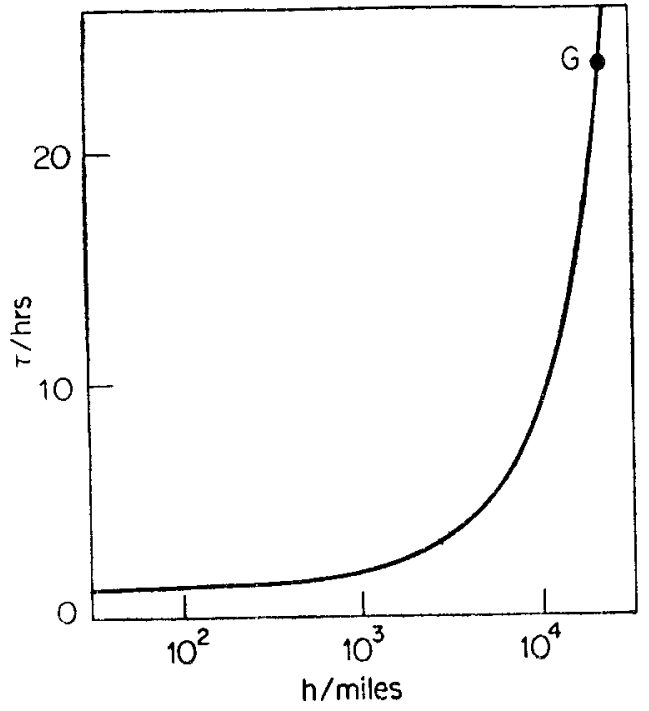

Figure 2. Orbital period $\tau$ as a function of orbital height $h$. The point $G$ represents the height at which a geostationary orbit, with $\tau=24$ hours, may be achieved.

with $\left(r_{0}+h\right)$ expressed in $\mathrm{km}$. Figure 2 shows orbital period as a function of height, and illustrates the fact that at heights below $1500 \mathrm{~km}$ the orbital period is roughly constant at between 90 and 110 minutes. We also see that the orbital pericd reaches 24 hours, the same as that of the earth, at a height of $36080 \mathrm{~km}$ or 22420 miles ; when the sense of rotation is the same as that of the earth, this yields the so-called 'geostationary' orbit. If such an arbit lies in the earth's equatorial plane, the object will appear stationary in the sky.

Orbital heights for Spacelab and similar missions are in the $150-300 \mathrm{~km}$ range, where atmospheric drag contributes a fairly steady residual acceleration of about $10^{-6} g_{0}$. Superimposed on this, short-term accelerations occur, random in both 
duration and direction, of about $10^{-4}$ to $10^{-6} \mathrm{~g}_{0}$, attributable in part to gas venting and attitude control thrusters, and also to crew movements in the case of manned missions.

If there were an experimental demand for even lower accelerations, it could be met, in principle, by allowing the experiment to flaat freely inside the spacecraft. If the latter were subject to a steady background acceleration of $10^{-6} \mathrm{~g}_{0}$, say, then the floating object would suffer an apparent displacement of $1 \mathrm{~m}$ in about $7 \frac{1}{2} \min$; however, this could be corrected by intermittent application of small rocket thrusts. Thus, the spacecraft could be driven round in a truly zero-gravity orbit by guiding it round a freely-floating body contained within itself. Since, however, most of the relevant fluid-mechanical dimensionless numbers involve the first power of $g$, and are therefore reduced by the factor $g / g_{0}$, effectively zerogravity conditions can be attained without going to such lengths.

Other types of space-flight pursue linear paths (ar, more strictly follow lines of gravitational force, normal to gravitational equipotential surfaces), such as interplanetary flights, or flights between earth and moon. These can be regarded as extended free fall, and so they also provide periods of weightlessness-though since they lie so far outside the earth's atmosphere, the residual acceleration due to viscous drag is altogether negligible. Once again, the major source of parasitic accelerations is shifts in the spacecraft centre of mass due to on-board movements.

\section{Plateau simulation}

For the study of certain types of capillary phenomenon, the value of $(\mathrm{B} 0)^{-1}$ can be made arbitrarily small by reducing $\Delta \rho$. It is interesting to note that when J A F Plateau, the Belgian physicist, developed this most elegant and visually attractive technique, he had been blind for about 30 years. In principle, pairs of immiscible liquids can be selected for which $\Delta \rho=0$ exactly, thou gh in practice, since such liquids will generally have different coefficients of thermal expansion, the condition will only be fulfilled at one temperature.

The pair of liquids used originally (Plateau 1873) (aqueous ethanol and olive oil) is inconvenient, because slow extraction of the alcohol into the oil phase causes a gradual diff of density. Our preferred recipe at Bristol uses pure water and an appropriate mixture of di-n-butyl phthalate and di-iso-octyl phthalate. The two esters are readily and cheaply available in technical grades (adequate for most purposes), with densities 1.043 and $0.980 \mathrm{gm} \mathrm{cm}^{-3}$ respectively at $20^{\circ} \mathrm{C}$. They form thermodynamically ideal mixtures over the whole composition range, so that given compositions can be made up in terms of volume or weight fractions without ambiguity, and their mutual solubility with water is almost zero. The following formula, based on measured values of thermal expansion of both organic and aqueous phases (Graham 1980) enables isodensity systems to be constructed to within $0.2 \%$ in the temperature range 20 to $28^{\circ} \mathrm{C}$

$$
V=V_{0}[1-\beta(25-T)] \text {, }
$$

where $V_{0}$ is the composition of the equal density mixture at $25^{\circ} \mathrm{C}(27.40 \%$ dibutyl phthalate by volume), $V$ is the composition of equal density at $T^{\circ} \mathrm{C}$ and the coefficient $\beta$ has the value 0.0410 in units of $\left({ }^{\circ} \mathrm{C}\right)^{-1}$. 
Plateau simulation is obviously a much cheaper way of reducing $\left(\mathbf{B}_{0}\right)^{-1}$ than any of those based on reducing $g$ itself. It offers an excellent means of studying the static stability of capillary configurations on a conveniently large scale and was used most productively in this way by Plateau himself and by many others since then (e.g. Mason 1970). However, it is not suitable for modelling dynamic phenomena-the rate of changes in interface shape is modified by the viscosity of the outer phase. Moreover internal convective motions take place in the normal terrestrial way since $\mathrm{Ra}$ and $\mathrm{Gr}$ are unaffected by making $\Delta \rho=0$. In addition, configurations of a fluid interface that are controlled by its angle of intersection (contact angle) with a solid surface are generally unsuited for study by Plateau's method, because the wetting behaviour in such systems is usually illdefined, and often shows large hysteresis effects.

It may be noted that close to a critical state, the density difference between two phases may tend to zero. However, this does not necessarily imply that $(\mathrm{Bo})^{-1}$ is becoming small, since the interfacial tension also tends to zero as the critical point is appraached. Moreover, since the compressibility of both phases tends to infinity a.t the same time, vertical gradients of density in the earth's gravitational field are magnified in the critical region. For the latter reason, a critical state can never be sharply defined in a system of finite dimensions in a gravitational field, and the study of critical phenomena in micro-gravity is therefore an important and promising field.

\section{References}

Boucher E A and Evans M J B 1975 Proc. R. Soc. (London) A346 349

Graham R M M 1980 unpublished work, Bristol

Mason G 1970 J. Colloid. Interface Sci. 32172

Plateau J A F 1873 Statique expérimentale et théorique des liquides soumis aux seules forces moléculaires, Gauthier-Villars 